\title{
Une sélection de ressources sur l'éducation au Maghreb
}

Cécile de Bouttemont

\section{OpenEdition}

1 Journals

Édition électronique

URL : https://journals.openedition.org/ries/671

DOI : 10.4000/ries.671

ISSN : 2261-4265

Éditeur

France Education international

Édition imprimée

Date de publication : 1 décembre 2009

Pagination : 16-18

ISBN : 978-2-8542-0577-0

ISSN : $1254-4590$

Référence électronique

Cécile de Bouttemont, "Une sélection de ressources sur l'éducation au Maghreb », Revue internationale d'éducation de Sèvres [En ligne], 52 I décembre 2009, mis en ligne le 01 décembre 2010, consulté le 27 avril 2022. URL : http://journals.openedition.org/ries/671 ; DOl : https://doi.org/10.4000/ries.671

Ce document a été généré automatiquement le 27 avril 2022.

(c) Tous droits réservés 


\title{
Une sélection de ressources sur l'éducation au Maghreb
}

\author{
Cécile de Bouttemont
}

1 L'article s'inscrit dans la continuité des ressources consacrées à l'éducation par zone géographique : l'Amérique latine, les Caraïbes ( $n^{\circ} 45$, septembre 2007), l'Asie ( ${ }^{\circ} 48$, septembre 2008) et l'Afrique subsaharienne ( $n^{\circ} 50$, avril 2009). Nous avons sélectionné des organismes internationaux, régionaux, ainsi que des institutions financières intervenant dans le domaine de l'éducation dans les pays du Maghreb. Les ressources présentées ici sont d'accès libre et gratuit.

2 Sitographie arrêtée le 15 septembre 2009.

\section{Organismes}

\section{Organismes internationaux}

Institut international de planification de l'éducation (IIPE)

3 Centre de formation et de recherche de l'Unesco spécialisé en planification et gestion de l'éducation, l'IIPE a pour objectif d'aider les pays à développer leurs systèmes éducatifs. Son site (en français, anglais et espagnol) donne accès à la liste des projets menés dans les États arabes en 2006-2007. Planipolis, portail des plans et des politiques d'éducation des États membres de l'IIPE, met en ligne des documents émanant de sources officielles par pays: politiques nationales d'éducation, plans nationaux d'éducation dont ceux pour l'éducation pour tous (EPT), rapports sur les objectifs pour le millénaire du développement (OMD). La recherche s'effectue par zone géographique, mot clé et type de document. http://www.iiep.unesco.org/fr/liipe-dans-le-monde/ etats-arabes.html 


\section{Bureau international d'éducation (BIE)}

4 Centre de l'Unesco spécialisé dans le développement des contenus éducatifs, le BIE met en ligne (en anglais, français, espagnol, russe, arabe, chinois) des informations sur les systèmes éducatifs et les curricula, les programmes, les projets et les activités en cours ; des ressources (rapports nationaux, dossiers par pays); et des bases de données. La rubrique " Dossiers pays ", (entrée par région ou par pays) propose des données sur les systèmes d'enseignement, les curricula et une sélection de liens vers des sources officielles d'information sur l'éducation de chacun des pays concernés. http:// www.ibe.unesco.org/fr/dans-le-monde/etats-arabes.html

\section{Association internationale des universités (AIU)}

5 Affiliée à l'Unesco, l'AIU regroupe les universités de près de cent cinquante pays afin de croiser réflexions et actions sur leurs préoccupations communes. Ses thématiques de travail concernent en particulier l'accès à l'enseignement supérieur et l'éducation pour tous, l'internationalisation et le dialogue interculturel. L'AIU tient à jour plusieurs bases de données dont une sur l'enseignement supérieur; les systèmes éducatifs et la liste des universités sont disponibles, par pays. http://www.unesco.org/iau/ onlinedatabases/fre/index.html

\section{Institut de statistique de l'Unesco (ISU)}

6 Il a pour mission de collecter, d'analyser, et diffuser les données statistiques dans les domaines d'intervention de l'Unesco au profit de la communauté internationale. Il publie des résumés et des études statistiques, des rapports de recherche. Parmi ses publications clés figure le Recueil de données mondiales sur l'éducation qui permet une lecture comparée des statistiques internationales. Le site propose des statistiques par pays et par niveau d'enseignement.

\section{Organismes régionaux}

\section{Bureau de Rabat}

7 Représentant l'Unesco auprès des cinq pays du Maghreb (Algérie, Maroc, Tunisie, Libye, Mauritanie), le bureau de Rabat est chargé d'apporter une assistance technique à la mise en place et à la réforme des politiques éducatives nationales afin que celles-ci répondent aux besoins réels et spécifiques de chacun des pays du Maghreb. L'amélioration de la qualité de l'enseignement est aussi l'une de ses priorités, le site propose la liste des projets, études, ressources globales sur les politiques d'éducation mises en œuvre dans cette région. Il donne également des fiches générales sur chacun des pays avec l'actualité éducative correspondante. http://rabat.unesco.org/

\section{Bureau Europe de l'Ouest et Maghreb de l'AUF}

8 Représentant de l'Agence universitaire de la Francophonie (AUF) en Europe de l'Ouest et au Maghreb, ce bureau a pour mandat de contribuer au développement de la communauté universitaire et scientifique francophone et d'appliquer les programmes d'action de l'AUF sur la mobilité des enseignants, des chercheurs et des étudiants. Il 
favorise les projets de coopération universitaire et scientifique avec des établissements d'enseignement supérieur et de recherche. Le site propose une actualité éducative sur la zone concernée avec plus particulièrement la situation de l'enseignement du français. Le site propose une série de publication concernant l'enseignement supérieur et la recherche. http://www.auf.org/regions/europe-ouest-maghreb/

\section{Organisation arabe pour l'éducation, la culture et la science (ALECSO)}

Réunissant 22 membres et basée à Tunis, cette organisation est chargée de développer la coopération régionale entre les organisations arabes opérant dans le domaine de l'éducation pour généraliser l'enseignement de base obligatoire et l'alphabétisation sous toutes ses formes, ainsi que l'enseignement des adultes. Elle élabore également des études scientifiques proposant des moyens de mises en œuvre, des sources de financement possibles en mettant à contribution des experts en vue d'aider les États à concevoir des stratégies. Plusieurs bases de données sont disponibles sur le site (uniquement en langue arabe), ainsi que les publications, les ateliers de travail et les séminaires. Le site propose quelques ressources en français comme les plans de développement de l'enseignement dans le monde arabe. http://www.alecso.org.tn/

\section{Institutions financières}

\section{Banque mondiale}

10 Organisme international, la Banque mondiale, apporte un appui financier et technique aux pays en développement. Elle soutient de nombreux projets, notamment en éducation. Ses publications, rapports (régionaux ou nationaux) ainsi que les données statistiques se déclinent par pays de la zone MENA (Middle East North Africa) en français, anglais et espagnol. La rubrique "Profils pays", donne accès à des données éducatives par pays (synthèses, indicateurs internationaux, statistiques) et permet de connaître les activités de la Banque mondiale dans une région ou un pays (projets/programmes, publications, rapports). www.banquemondiale.org/

\section{Groupe de la Banque africaine de développement (BAD)}

11 La BAD est une banque de développement régionale et multilatérale dont le principal objectif est de promouvoir une croissance économique durable et de réduire la pauvreté en Afrique. Elle finance un large éventail de projets et programmes nationaux et multinationaux, dont certains axes concernent le secteur éducatif. L'entrée Afrique du Nord (français, anglais), permet d'accéder aux documents clés d'un pays de la zone du Maghreb (documents de stratégies pays, NEPAD, etc.) ainsi qu'à une sélection de liens. La rubrique « Documents », donne accès, par pays et par thématique, à d'autres informations (rapports, projets). www.afdb.org/ 
INDEX

Mots-clés : politique éducative

Index géographique : Maghreb, Algérie, Tunisie, Maroc, Mauritanie, Libye

Keywords : educational policy

Palabras claves : política educacional

\section{AUTEUR}

\section{CÉCILE DE BOUTTEMONT}

Documentaliste au centre de ressources en ingénierie documentaire du CIEP 